This item was submitted to Loughborough's Research Repository by the author.

Items in Figshare are protected by copyright, with all rights reserved, unless otherwise indicated.

\title{
Performance of Bayesian estimation based variable rate variable power MQAM system
}

PLEASE CITE THE PUBLISHED VERSION

PUBLISHER

(c) IEEE

VERSION

VoR (Version of Record)

\section{LICENCE}

CC BY-NC-ND 4.0

\section{REPOSITORY RECORD}

Ong, Lay Teen, Sangarapillai Lambotharan, Jonathon Chambers, and Mohammad Shikh-Bahaei. 2019. "Performance of Bayesian Estimation Based Variable Rate Variable Power MQAM System". figshare. https://hdl.handle.net/2134/5542. 
This item was submitted to Loughborough's Institutional Repository (https://dspace.lboro.ac.uk/) by the author and is made available under the following Creative Commons Licence conditions.

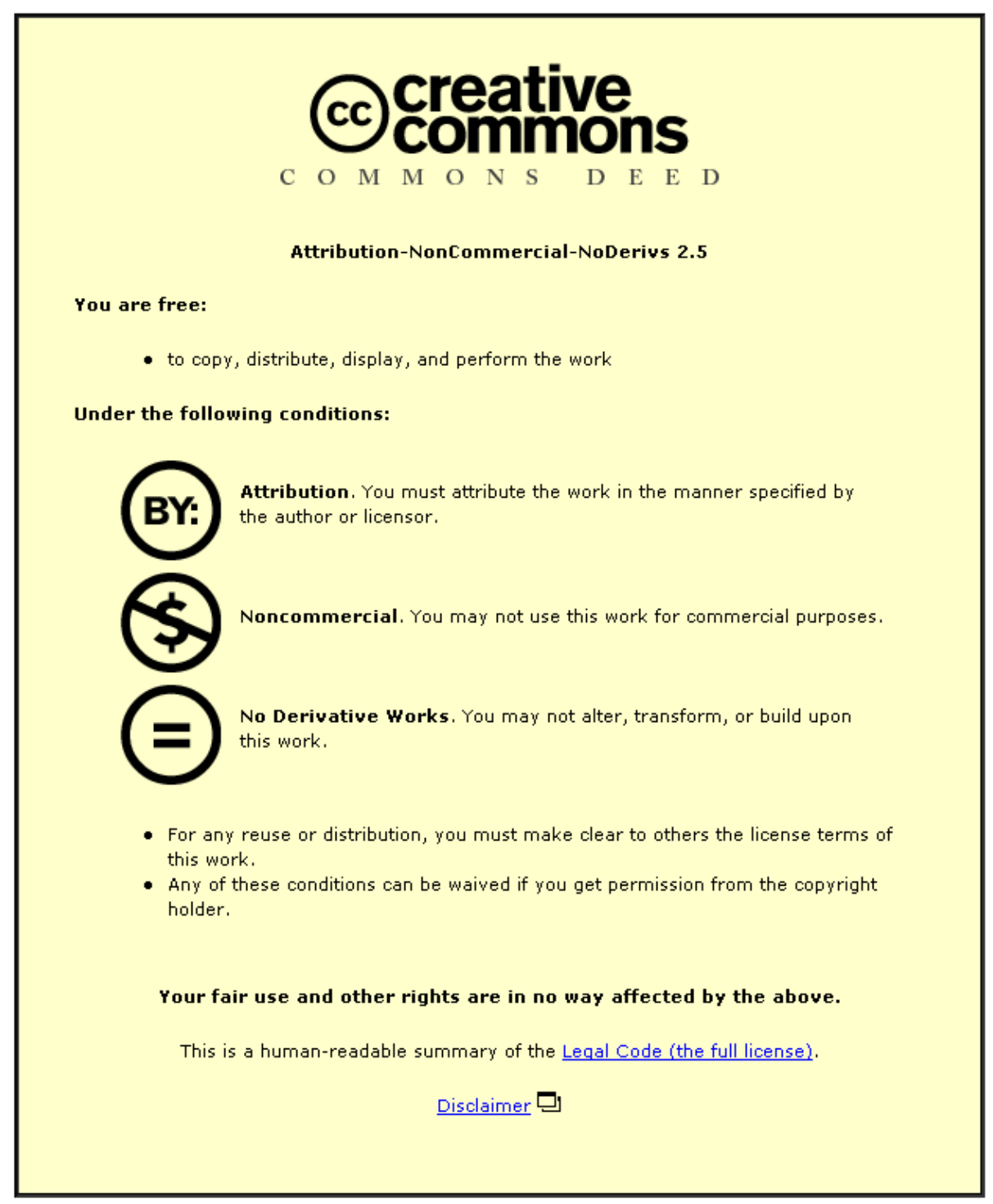

For the full text of this licence, please go to: http://creativecommons.org/licenses/by-nc-nd/2.5/ 


\section{PERFORMANCE OF BAYESIAN ESTIMATION BASED VARIABLE RATE VARIABLE POWER MQAM SYSTEM}

\author{
L. T. Ong, S. Lambotharan, J. A. Chambers \\ Centre of Digital Signal Processing \\ Cardiff University \\ Cardiff CF24 0YF, UK
}

\author{
M. Shikh-Bahaei \\ Department of Electronic Engineering \\ King's College London \\ London WC2R 2LS, UK
}

\begin{abstract}
In this paper, we generalize the algorithms of our previously proposed Bayesian estimation based variable rate variable power multilevel quadrature amplitude modulation (VRVPMQAM) system to incorporate for the first time a maximum a posteriori (MAP) channel predictor and a MQAM scheme adopted with practical constellation sizes. Based on a pilot symbol assisted modulation (PSAM) scheme, we evaluate the performance of our proposed VRVP-MQAM system over a Rayleigh flat-fading channel. We demonstrate in our simulation results that the proposed rate and power algorithms that are derived based on a Bayesian bit error rate (BER) estimation and the second order statistical characterization of the channel state information (CSI) outperforms in terms of spectral efficiency and average BER. This improvement is confirmed by comparison with an alternative rate and power algorithm which exploits an ideal CSI assumption.
\end{abstract}

\section{INTRODUCTION}

To enhance spectral efficiency of a wireless communication system over a fading channel, transmission parameters such as modulation, code rate and transmitted power signal should be adjusted to adapt to the varying nature of the channel [1-3]. Using these optimal techniques, it is possible to attain Shannon capacity when perfect channel state information (CSI) is available at the transmitter [3]. Imperfect CSI is known to degrade the performance of an adaptive system. Imperfect CSI can be resulted from estimation error introduced in the receiver, delay and error in the feedback of CSI to the transmitter [4-6]. For example, [4] has proposed a variable-rate and variable-power uncoded MQAM (VRVP-MQAM) system that achieves higher power gain over a fixed-rate and variable power system. However the work in [4] is based on a perfect CSI assumption, and therefore it is sensitive to channel estimation and feedback errors. [4] had showed the impact of the channel estimation error and delay on bit error rate (BER) performance through analytical evaluation. [5] extended the VRVP-MQAM algorithm based on a pilot symbol assisted modulation (PSAM) method, and derived a new set of analytical formulas embedding imperfect channel estimates and feedback delay parameters. On the other hand, [6] investigated the impact of CSI imperfections based on channel prediction of a fixed power, adaptive coded modulation (ACM) system. For optimal ACM performance, [6] considered a PSAM method for coherent detection and a maximum a posteriori-optimal (MAP) envelope predictor for channel prediction. The work in [4-6] produced analytical expression to investigate the impact of delay in feedback channel on the BER performance.

In this work, we study the Bayesian estimation based VRVPMQAM scheme that we derived based on the second order statistical characterization of the channel state imperfection over a Rayleigh flat-fading channel [7]. We will compare through simulation results, the performance of our VRVP-MQAM algorithm to an alternate VRVP-MQAM algorithm that is based on perfect CSI assumption. We will employ a practical MQAM scheme and use the Jakes fading model [8].

The rest of the paper is orgainzed as follows. Section II presents the system model. Section III discusses the adaptive rate and power algorithms. In section IV, the channel predictor is presented. Section V describes the simulation parameters and analyses the simulation results. Finally conclusions are drawn in section VI.

\section{SySTEM MODEL}

We consider an uncoded adaptive modulation and power MQAM system over a Rayleigh flat-fading channel. Fig. 1 depicts the block diagram of the system. We denote the transmitted complex baseband signal as $x(i)$, where $i$ denotes the time index. We consider a PSAM scheme [9] with the frame structure shown in Fig. 2. We assume that pilot symbol is inserted at the first symbol location of each data slot and is repeated periodically at $L$ symbols interval. Therefore we can represent the pilot symbol as $x(i-k L)$, where $k \in\{0,1,2, \ldots\}$. We also assume that the power of the pilot signal is equal to the average data signal power. At the receiver, after matched filtering and sampling with perfect symbol timing at a rate of $1 / T_{s}$, where $T_{s}$ is the symbol duration, the received complex-valued signal over a flat-fading channel can be written as

$$
y(i)=z(i) x(i)+n(i),
$$

where $z(i)$ denotes the complex fading and $n(i)$ is circularly symmetric complex additive white Gaussian noise (AWGN). The noise variance is defined as $\sigma_{n}^{2}=\frac{N_{O}}{2}$, where $N_{0}$ is the noise power spectral density. We shall assume perfect signal detection at the receiver using pilot symbols. The instantaneous received signal-to-noise ratio (SNR) based on the predicted fading $\breve{z}(i)$, is defined as $\breve{\gamma}(i)=\bar{S}|\breve{z}(i)|^{2} /\left(N_{0} B\right)$, where $\bar{S}$ denotes the average transmit signal power and $B$ denotes the received signal bandwidth. Note that the actual instantaneous SNR based on the fading channel $z(i)$ is $\gamma(i)=$ $\bar{S}|z(i)|^{2} /\left(N_{0} B\right)$. The average received SNR is $\Gamma=E[\gamma(i)]$ 


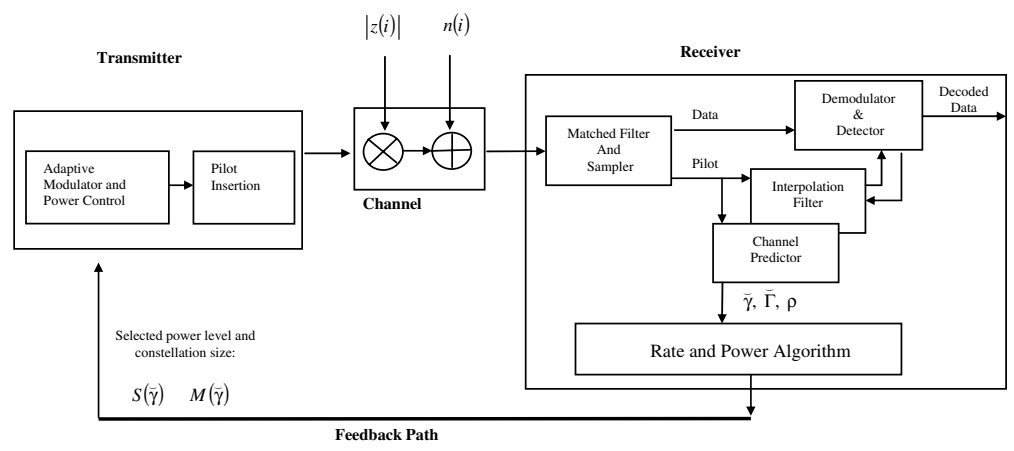

Figure 1: Block diagram of the proposed variable rate variable power MQAM system.

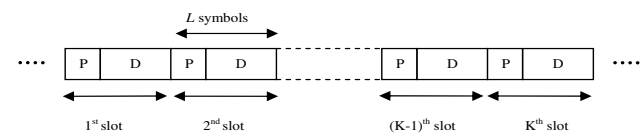

Figure 2: Frame structure: 'P' denotes pilot symbol, 'D' denotes data symbols and ' $\mathrm{K}$ ' defines the number of fading observations used in the channel predictor.

and its predicted value is $\breve{\Gamma}=E[\breve{\gamma}(i)]$, where $E[$.$] denotes$ the statistical expectation operator. Based on $\breve{\gamma}(i)$, the transmitter updates its rate and power by adjusting respectively its constellation size $M(\breve{\gamma})$ and power $S(\breve{\gamma})$. We consider that $M(\breve{\gamma})$ and $S(\breve{\gamma})$ are computed at the receiver and fedback as CSI to the transmitter. Consequently, by periodically updating its modulation and power level based on the feedback information, the transmitter would be able to adapt to a varying channel conditions. The performance of such link prediction using CSI feedback varies with the channel Doppler rate. In a channel prediction context, the performance can be parameterized by the error variance of the channel predictor at the receiver, feedback delay in CSI, and the update rate at the transmitter (i.e. how frequent transmitter updates its power and modulation level). As we are interested in the impact of delayed CSI on our proposed rate and power adaptation, we have considered here an optimal predictor with minimum error variance: a Rayleigh fading envelope predictor that is based on a MAP-optimal solution. The error variance of this MAP-optima predictor has been studied and verified in [6]. For a particular Doppler rate, we select the coefficients of the predictor that give very small error variance and would not degrade the system performance significantly. We shall also select the predictor coefficients such that the transmitter update rate is higher than the Doppler rate of the channel.

\section{ADAPTIVE RATE AND POWER ALGORITHM}

The transmitter will update its rate and power based on an instantaneous predicted SNR $\breve{\gamma}(i)$. For brevity, we shall drop the time index $i$ in the subsequent sections. We will investigate the performance of an adaptive rate and power algorithm that relates to the second order statistical characterization of the channel state imperfection, which was proposed in [7]. We denote this system as 'VRVP-CSI' scheme. We will compare the performance of this algorithm with adaptive rate and power algorithms based on a perfect CSI assumption [4]. We denote this system as 'VRVP-IDEAL' scheme. We shall give brief discussion of the work in [7] and [4]. We will use superscripts ' $C$ ' and ' $I$ ' to differentiate the algorithms for VRVP-CSI and VRVP-IDEAL schemes respectively. We will leave out the superscripts if the expression is applicable to both schemes.

The spectral efficiency of an MQAM scheme is defined as its average data rate per unit bandwidth. With data sent at $k(\breve{\gamma})=\log _{2}(M(\breve{\gamma}))$ bits/symbol, the instantaneous data rate is $k(\breve{\gamma}) / T_{s}$ bits/sec, where $T_{s}$ is the symbol time. Assuming Nyquist data pulses of duration $T_{s}=1 / B$, the spectral efficiency for the continous rate can be expressed as

$$
E[k(\breve{\gamma})]=\int_{\breve{\gamma}} k(\breve{\gamma}) f(\breve{\gamma}) d \breve{\gamma} \quad \text { bits/sce/Hz, }
$$

where $f(\breve{\gamma})$ denotes the probability density function (PDF) of $\breve{\gamma}$. Rate and power algorithms in [7] and [4] were derived for maximum spectral efficiency subject to an average power constraint

$$
E[S(\breve{\gamma})]=\int_{\breve{\gamma}} S(\breve{\gamma}) f(\breve{\gamma}) d \breve{\gamma} \leq \bar{S}
$$

and an instantaneous BER target (BERT) requirement. To solve the constrained optimization problem, the following Lagrangian equation is used.

$$
F(S(\breve{\gamma}))=\int_{\breve{\gamma}} k(\breve{\gamma}) f(\breve{\gamma}) d \breve{\gamma}+\lambda\left[\int_{\breve{\gamma}} S(\breve{\gamma}) f(\breve{\gamma}) d \breve{\gamma}-\bar{S}\right]
$$

where $\lambda$ denotes the Lagrange multiplier. Optimal solution for power adaptation of [7] and [4] were derived from the solution of $\frac{\partial F}{\partial S(.)}$.

\section{A. VRVP-CSI scheme}

In this paper, we generalize the algorithms of [7] to incoporate the MAP-optimal linear predictor [6], and represent the second order statistical characterization of the channel state imperfection through: i) $\Gamma$, ii) $\breve{\Gamma}$ and iii) $\rho$, the correlation coefficient between the predicted SNR $\breve{\gamma}$ and its true value $\gamma$.

Based on the observations of $\breve{\gamma}$, a Bayesian BER estimator was first derived using a MAP approach. By Bayes' theorem 
[10], the conditional PDF $f\left(p_{B} \mid \breve{\gamma}\right)$ is given by

$$
f\left(p_{B} \mid \breve{\gamma}\right)=\frac{f\left(\breve{\gamma} \mid p_{B}\right) f\left(p_{B}\right)}{f(\breve{\gamma})},
$$

where $p_{B}$ denotes the instantaneous BER, $f\left(p_{B}\right)$ denotes the probability density function (PDF) of $p_{B} . f\left(\breve{\gamma} \mid p_{B}\right)$ is the conditional PDF of $\breve{\gamma}$ given $p_{B}$. Since $f(\breve{\gamma})$ is a normalization factor, (5) is simplified to a final form of the MAP equation,

$$
l_{M A P}\left(p_{B}\right)=f\left(p_{B}, \breve{\gamma}\right),
$$

where $f\left(p_{B}, \breve{\gamma}\right)$ is the joint PDF of $p_{B}$ and $\breve{\gamma}$. We obtained $f\left(p_{B}, \breve{\gamma}\right)$ through the operation of random variables transformation using i) the BER expression of an MQAM scheme that is based on $\gamma$ and $\breve{\gamma}$ [4, eqn (42)],

$$
p_{B}(\gamma, \breve{\gamma})=c_{1} \exp \left(-\frac{c_{2} \gamma}{M(\breve{\gamma})-1} \frac{S(\breve{\gamma})}{\bar{S}}\right)
$$

where $c_{1}=0.2$ and $c_{2}=1.5$ [4]; and ii) the joint PDF of $\gamma$ and $\breve{\gamma}$ for PSAM method [11], denoted as $f(\gamma, \breve{\gamma})$,

$$
\begin{aligned}
f(\gamma, \breve{\gamma})= & \frac{1}{(1-\rho) \Gamma \hat{\Gamma}} I_{0}\left(\frac{2 \sqrt{\rho}}{1-\rho} \sqrt{\frac{\gamma \breve{\gamma}}{\Gamma \breve{\Gamma}}}\right) \times \\
& \exp \left(-\frac{1}{(1-\rho)}\left(\frac{\gamma}{\Gamma}+\frac{\breve{\gamma}}{\breve{\Gamma}}\right)\right) u(\gamma) u(\breve{\gamma}),
\end{aligned}
$$

where $I_{0}($.$) is the zero order modified Bessel function and u($. is the unit step function. Finally, the BER estimator was obtained from the solution of $\frac{\partial l_{M A P}}{\partial p_{B}}=0$, the result shown here as [7]

$$
\hat{p}_{B}(\breve{\gamma})=c_{1} \exp \left(\frac{\ln \left(\mathrm{BERT} / c_{1}\right) \rho \Gamma / \breve{\Gamma}}{\left(-\ln \left(\mathrm{BERT} / c_{1}\right) \Gamma / \breve{\gamma}(1-\rho)-1\right)^{2}}\right) .
$$

Based on $\breve{\gamma}$ and $\hat{p}_{B}$, we exploit the generic approximate BER expression [4, eqns (19-20)], and adapt rate through adjusting the constellation size $M(\breve{\gamma})$ as

$$
M^{C}(\breve{\gamma})=1+\frac{c_{2}}{-\ln \left(\hat{p}_{B}(\breve{\gamma}) / c_{1}\right)} \frac{S(\breve{\gamma})^{C}}{S} \breve{\gamma}
$$

Substituting (9) into (10), the final form of $M^{C}(\breve{\gamma})$ is obtained as

$$
M^{C}(\breve{\gamma})=1+\frac{K \breve{\Gamma}}{\rho \Gamma}\left\{\frac{c_{2} \Gamma}{K \breve{\gamma}}(1-\rho)-1\right\}^{2} \frac{S(\breve{\gamma})^{C}}{S} \breve{\gamma}
$$

where $K=\frac{c_{2}}{-\ln \left(\frac{\mathrm{BERT}}{c_{1}}\right)} \cdot \frac{S(\breve{\gamma})}{S}{ }^{C}$ is the solution to the optimal power adaptation, expressed as [7, c.f. eqn(21)]

$$
\frac{S(\breve{\gamma})}{\bar{S}}^{C}= \begin{cases}U^{C}-\frac{\frac{\Gamma}{\grave{\Gamma}} \rho}{K \breve{\gamma}\left\{\frac{c_{2}}{K} \bar{\gamma}(1-\rho)-1\right\}^{2}}, & S(\breve{\gamma}) \geq 0, \\ 0, & \text { otherwise, }\end{cases}
$$

$U^{C}$ is a constant value found through numerical search such that average power constraint (3) is satisfied. When $S(\breve{\gamma})^{C}<$ 0 , no data is transmitted.

\section{B. VRVP-IDEAL scheme}

To compare to the VRVP-IDEAL scheme, we use the same channel predictor and the discrete level MQAM approach. We invoke rate adaptation through adjusting the constellation size $M(\breve{\gamma})$ using [4, c.f. eqn. (20)]

$$
M^{I}(\breve{\gamma})=1+K \frac{S(\breve{\gamma})}{S}^{I} \breve{\gamma}
$$

where $\frac{S(\breve{\gamma})}{S}^{I}$ is the power adaptation [4, c.f. eqn. (23)] given as

$$
\frac{S(\breve{\gamma})^{I}}{\bar{S}}= \begin{cases}U^{I}-\frac{1}{\breve{\gamma}}, & S(\breve{\gamma}) \geq 0 \\ 0, & \text { otherwise. }\end{cases}
$$

$U^{I}$ is a constant value found through numerical search such that average power constraint (3) is satisfied. When $S(\breve{\gamma})^{I}<0$, no data is transmitted. Since VRVP-IDEAL scheme is based on an ideal CSI assumption, we note that unlike (11) and (12), the algorithms in (13) and (14) do not have parameters that relate to the channel state imperfections.

\section{Spectral Efficiency and Average BER}

To consider a practical constellation size for MQAM system, we adopt a discrete rate adaptation with $M$ restricted to a positive integer value that can be represented by a factor of 2 . Moreover, with the transceiver design complexity growth as the constellation size increases, there is a limit to the maximum constellation size used. Let $G$ denote the number of modulation levels considered for the system, the adaptive modulation level will be chosen from the set $\left\{M_{d}\right\}_{d=0}^{G-1}$, where element $M_{d}$ can be $\{0,2,4,8 \ldots\}$. Note that $M_{d}=0$ is assigned to the truncated channel situation where no data transmission is allowed. Since the computed value of $M(\breve{\gamma})$ is a real number, we will choose the largest possible discrete $M_{d}$ value that is smaller than or equal to $M(\breve{\gamma})$ to ensure a BER lower than the BERT requirement.

The discrete transmission rate associated with each $M_{d}$ is $k_{d} / T_{s}$ bits/sec, where $k_{d}=\log _{2}\left(M_{d}\right)$ bits/symbol. The spectral efficiency for the discrete-rate approach can be expressed as

$$
\frac{R}{B}=\sum_{d=1}^{G-1} k_{d}\left(\frac{L-1}{L}\right) P_{d} \quad \text { bits } / \mathrm{sec} / \mathrm{Hz}
$$

where $P_{d}$ is the probability that the modulation level $M_{d}$ will be used. The term $(L-1) / L$ corresponds to an efficiency factor of the PSAM scheme; since pilot symbol is used as control information and consumes a small ratio of the actual data capacity.

The average BER can be expressed as

$$
\overline{B E R}=\frac{\sum_{d=1}^{G-1} k_{d} \overline{B E R}_{d}}{R / B},
$$

where $\overline{B E R}_{d}$ is the average BER experienced in each discrete rate $k_{d}$. We note the discrete $M_{d}$ level selection will result in a lower $\overline{B E R}_{d}$ performance than the required BERT. This is due to the fact that $M_{d}$ is chosen to be less than or equal to $M(\breve{\gamma})$. 
However we shall emphasis that channel imperfections will degrade the average BER performance. We will demonstrate the impact through system simulation in section V.

\section{Channel Prediction}

At the receiver, based on the knowledge of the pilot symbols, the channel fading at the pilot symbol instants can be represented as

$$
\begin{aligned}
\hat{z}(v-k L) & =\frac{y(v-k L)}{x(v-k L)} \\
& =z(v-k L)+\frac{n(v-k L)}{x(v-k L)}
\end{aligned}
$$

$(v-k L)$ denotes the time instant of a pilot symbol, where $v=p L, p \in \mathbb{Z}$ and $k=0,1, \ldots, K . K$ defines the number of fading observations used in the channel predictor. In sections II and III, we introduced that the transmitter updates its rate and power at time instant $i$. For a delayed feedback path, we can define $i=v+j$, where $j=q L$ and $q \in\{1,2, \ldots\}$. Therefore, with time instant $j$ used to associate time delay parameter, $\breve{z}(i)=\breve{z}(v+j)$. For a linear predictor of order $K$, the predicted fading channel using $\mathrm{K}$ fading estimates $\hat{z}(v-k L)$ can be expressed as

$$
\begin{aligned}
\breve{z}(v+j) & =\sum_{k=0}^{K-1} f_{j}^{*}(k) \hat{z}(v-k L) \\
& =\mathbf{f}_{j}^{H} \mathbf{z}_{v},
\end{aligned}
$$

where $\mathbf{f}_{j}^{H}=\left[f_{j}^{*}(0), f_{j}^{*}(1), \ldots, f_{j}^{*}(K-1)\right]$ is the predictor filter coefficient vector, ${ }^{*}$ denotes a conjugate funtion and $\mathbf{z}_{v}=[\hat{z}(v), \hat{z}(v-L), \ldots, \hat{z}(v-(K-1) L)]^{T}$ is the vector of the estimates of the complex fading amplitude in the last $\mathrm{K}$ pilot-symbol instants. To evaluate the performance in terms of the optimality of a system, we have adopted the MAP-optimal envelope prediction solution, which is obtained and demonstrated in [6] (and references within) as one of the best linear predictor (in an mean-square error sense) applicable for Rayleigh fading. It is shown in [6] that the MAP-optimal prediction filter coefficient vector is expressed as

$$
\mathbf{f}_{j, \mathrm{MAP}}^{T}=\mathbf{r}_{j}^{T}\left(\mathbf{R}+\frac{1}{\hat{\gamma}} \mathbf{I}\right)^{-1}
$$

where $\mathbf{r}_{j}^{T}$ is a vector whose elements $r_{j, k}$ are the correlation between the fading at the pilot-symbol instants and the fading at the prediction time $v+j . \mathbf{R}$ is a $K \mathrm{x} K$ covariance matrix whose elements $R_{k, l}$ are the correlation of the fading between pilot-symbol instants. Elements of $\mathbf{r}_{j}^{T}$ and $\mathbf{R}$ can be expressed in terms of the normalized correlation function $R($.$) :$

$$
\begin{aligned}
r_{j, k} & =\frac{1}{\Omega} E[z(v+j) z(v-k L)] \\
& =R\left((j+k L) T_{s}\right), \\
R_{k, l} & =R\left((|k-l|) L T_{s}\right),
\end{aligned}
$$

where $\Omega=E\left[|z|^{2}\right]$ and $|k-l|$ is the time difference between fading at two pilot symbols. When the standard Jakes model is used, $R($.$) can be expressed in terms of the Bessel function$ $R(\tau)=J_{o}\left(2 \pi f_{d} \tau\right)$, where $J_{o}($.$) is the zeroth-order Bessel$ function of the first kind and $\tau$ denotes a time unit argument. $f_{d}$ is the Doppler spread defined by $f v_{t} / c$, where $f$ is the carrier frequency $(\mathrm{Hz}), v_{t}$ is the terminal speed $(\mathrm{m} / \mathrm{sec})$ and $c$ is the speed of light $(\mathrm{m} / \mathrm{sec})$.

Finally, with the MAP-optimal predictor, the correlation coefficient $\rho$ is equal to [6],

$$
\rho=\mathbf{r}_{j}^{T}\left(\mathbf{R}+\frac{1}{\hat{\gamma}} \mathbf{I}\right)^{-1} \mathbf{r}_{j}
$$

\section{Simulation Results}

We adopt the modified Jakes model [8] in our simulation, which produces uncorrelated fading waveforms that matches the theoretical Jakes model function. An uncorrelated fading waveform with real and imaginary parts having equal power is represented as [8]

$$
T(t)=\sqrt{\frac{2}{N_{o s c}} \sum_{n=1}^{N_{o s c}}\left[\cos \left(\beta_{n}\right)+I \sin \left(\beta_{n}\right)\right] \cos \left(\omega_{n} t+\theta_{n}\right)},
$$

where $t$ is the time samples, $I$ denotes $\sqrt{-1}$ and $N_{\text {osc }}$ denotes the number of oscillators associated to the reflected rays arrive at the receiver. $\beta_{n}$ are the phases of the oscillators expressed as $\pi n / N_{\text {osc }}$ and $\theta_{n}$ are the initial phases of the oscillators. Each rays experiences a Doppler spread defined by $\omega_{n}=\omega_{M} \cos \left(\alpha_{n}\right)$, where $\omega_{M}=2 \pi f v_{t} / c$. The rays arrival angles $\alpha_{n}$ is defined as $2 \pi(n-0.5) / N_{\text {osc }}$.

Based on this reformulated Jakes model, we generate complex fading waveform using $N_{\text {osc }}=16$. We use a normalized doppler spread $f_{d} T_{s}$ of 0.001 . We set the carrier frequency $f=2 \mathrm{GHz}$, transmission bandwidth $B=200 \mathrm{kHz}$ with Nyquist sampling and thus symbol duration $T_{s}=1 / B$ sec. The terminal velocity is $v_{t}=30 \mathrm{~m} / \mathrm{sec}$. For minimum prediction error variance, the optimal MAP predictor is set to $L=10$ and $K=500$. We set $M_{D}$ to $(0,2,4,16,64)$.

We show in Fig. 3 and Fig. 4 simulation results for spectral efficiency and average BER performance over a set of normalized time delay parameters $f_{d} T_{s} j$ (Table 1 ) at $\Gamma=15 \mathrm{~dB}$ and $\Gamma=20 \mathrm{~dB}$. Table 1 also illustrates the value of $f_{d} T_{s} j$ to its equivalent delay in symbol instants, and its associate CSI parameter $\rho$. The VRVP-CSI system outperforms in spectral efficiency when compared to the alternate system VRVP-IDEAL . Fig. 3 demonstrates that the VRVP-CSI system experiences a higher spectral efficiency gain across the normalized time delay for a higher average SNR $(\sim 0.15 \mathrm{bps} / \mathrm{Hz}$ gain at $\Gamma=20 \mathrm{~dB}$ and compared to $\sim 0.05 \mathrm{bps} / \mathrm{Hz}$ gain at $\Gamma=15 \mathrm{~dB}$ ). In terms of average BER performance, the simulation results in Fig. 4 show several interesting points. Firstly, we note that both systems perform better than the BERT threshold at a lower feedback delay. This is because the adaptive modulator uses a largest possible discrete $M_{d}$ which is below or equal to the actual optimal M (refer to the details discussed in section III). Secondly, we note that our VRVP-CSI algorithm tolerates a higher feedback delay before the BER performance increases beyond 


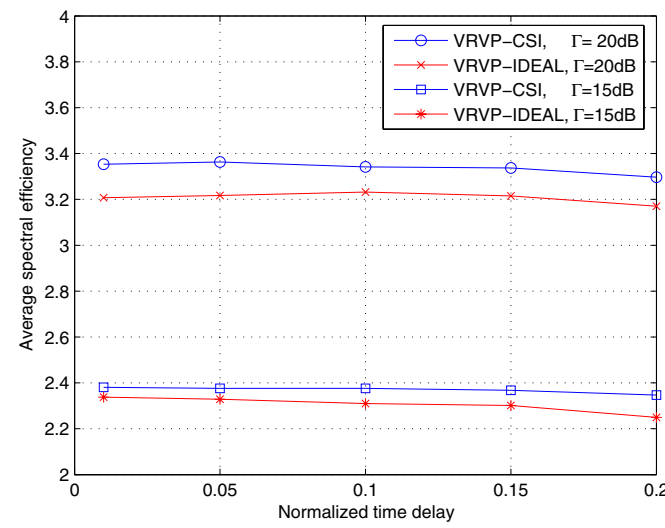

Figure 3: Average spectral efficiency (bits/sec/Hz) versus normalized time delay $f_{d} T_{s} j, \Gamma=15 \mathrm{~dB}, 20 \mathrm{~dB}$ and BERT $=10^{-3}$.

Table 1: Values of correlation coefficient $\rho$ and delay at $f_{d} T_{s}=0.001, \Gamma=15 \mathrm{~dB}, 20 \mathrm{~dB} . f_{d} T_{s} j$ denotes normalized time delay and $q L$ denotes delay in symbol units.

\begin{tabular}{|l|l|l|l|}
\hline$f_{d} T_{s} j$ & $q L$ & $\Gamma=20 \mathrm{~dB}$ & $\Gamma=15 \mathrm{~dB}$ \\
\cline { 3 - 4 } & (symbols) & $\rho$ & $\rho$ \\
\hline 0.01 & 10 & 0.9981 & 0.9948 \\
\hline 0.05 & 50 & 0.9961 & 0.9906 \\
\hline 0.1 & 100 & 0.9915 & 0.9831 \\
\hline 0.15 & 150 & 0.9831 & 0.966 \\
\hline 0.2 & 200 & 0.9692 & 0.9426 \\
\hline
\end{tabular}

the BERT threshold. For instance, at $\Gamma=15 \mathrm{~dB}$, VRVP-CSI scheme shown in Fig. 4 takes approximately 180 symbols delay before its average BER increases beyond the BERT $\left(10^{-3}\right)$ requirement while the VRVP-IDEAL scheme allows for only 150 symbols delay.

\section{CONCLUSION}

We have demonstrated the impact of feedback delay to channel prediction on an uncoded VRVP-MQAM system based on Jakes fading simulation model. The system simulation was evaluated based on a PSAM approach and a Rayleigh fading envelope channel predictor. We proposed a Bayesian based adaptive rate and power algorithm, denoted as VRVPCSI scheme, that adapts rate and power in relation to the second order statistical characterization of the channel state imperfection. We compared the performance to an alternate system with rate and power algorithms derived based on a perfect CSI assumption. We denote the second set of algorithm as VRVPIDEAL scheme. We showed in our simulation results that our proposed VRVP-CSI based system outperforms in terms of spectral efficiency when compared to the VRVP-IDEAL based system. In terms of BER performance, we demonstrated that both VRVP-CSI and VRVP-IDEAL schemes that use practical discrete M-level selection can tolerate a small delay in CSI feedback before the average BER goes beyond the BER target requirement. Furthermore the simulated BER results showed that our proposed VRVP-CSI scheme is less sensitive to feedback delay as compared to the VRVP-IDEAL scheme.
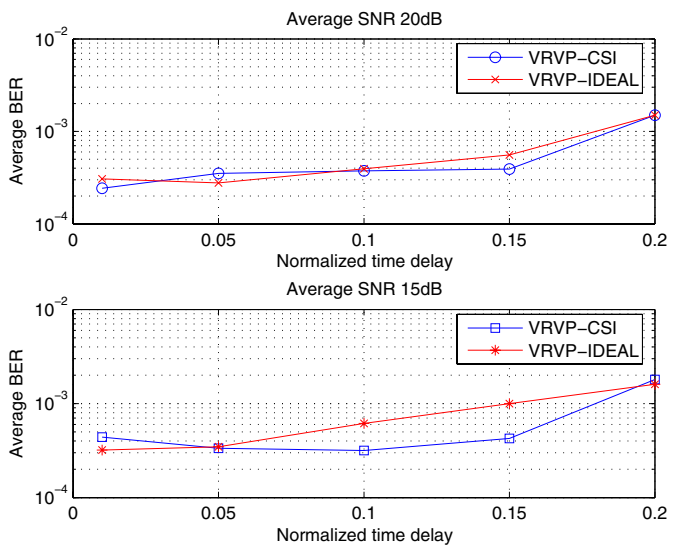

Figure 4: Average BER versus normalized time delay $f_{d} T_{s} j$, $\Gamma=15 \mathrm{~dB}, 20 \mathrm{~dB}$ and $\mathrm{BERT}=10^{-3}$.

\section{REFERENCES}

[1] E. Biglieri, J. Proakis, and S. Shamai, "Fading channels: informationtheoretic and communications aspects," IEEE Trans. Info. Theory, vol. 44, pp. 2619 - 2692, Oct. 1998.

[2] D. L. Goeckel, "Adaptive coding for time-varying channels using outdated fading estimates," IEEE Trans. Commun., vol. 47, pp. $844-855$, Jun. 1999.

[3] A. J. Goldsmith and P. P. Varaiya, "Capacity of fading channels with channel side information," IEEE Trans. Info. Theory, vol. 43, pp. 19861992, Nov. 1997.

[4] A. J. Goldsmith and S. Chua, "Variable-rate variable-power MQAM for fading channels," IEEE Trans. Commun., vol. 45, pp. 1218 -1230, Oct. 1997.

[5] J. F. Paris, M. C. Aguayo-Torres, and J. T. Entrambasaguas, "Impact of channel estimation error on adaptive modulation performance in flat fading," IEEE Trans. Commun., vol. 52, pp. 716 -720, May. 2004.

[6] G. E. Oien, H. Holm, and K. J. Hole, "Impact of channel prediction on adaptive coded modulation performance in Rayleigh fading," IEEE Trans. Veh. Technol., vol. 53, pp. 758 - 769, May 2004.

[7] L. T. Ong, S. Lambotharan, M. Shikh-Bahaei, and J. A. Chambers, "Variable rate and variable power MQAM system based on Bayesian bit error rate and channel estimation techniques," Submitted to IEEE Trans. Commun., March 2006.

[8] P. Dent, G. Bottomley, and T. Croft, "Jakes fading model revisited," Electronics Letters, vol. 29, pp. 1162 - 1163, Jun. 1993.

[9] J. K. Cavers, "An analysis of pilot symbol assisted modulation for Rayleigh fading channels," IEEE Trans. Veh. Technol., vol. 40, pp. $686-$ 693, Nov. 1991.

[10] A. Papoulis, Probability, Random and Stochastic Process. McGraw-Hill, 1984.

[11] X. Tang, M.-S. Alouini, and A. J. Goldsmith, "Effect of channel estimation error on M-QAM BER performance in rayleigh fading," IEEE Trans. Commun., vol. 47, pp. 1856 - 1864, Dec. 1999. 